\title{
USEFULNESS OF SEQUENTIAL ORGAN FAILURE ASSESSMENT (SOFA) AND ACUTE PHYSIOLOGY AND CHRONIC HEALTH EVALUATION II (APACHE II) SCORE IN ANALYSING PATIENTS WITH MULTIPLE ORGAN DYSFUNCTION SYNDROME IN SEPSIS
}

\author{
K.S. Abhinandan ${ }^{1}$, R. Vedavathi²
}

\section{HOW TO CITE THIS ARTICLE:}

K.S. Abhinandan, R. Vedavathi. "Usefulness of sequential organ failure assessment (sofa) and acute physiology and chronic health evaluation II) score in analysing patients with multiple organ dysfunction syndrome in sepsis". Journal of Evolution of Medical and Dental Sciences 2013; Vol2, Issue 49, December 09; Page: 9591-9605.

ABSTRACT: BACKGROUND: Sepsis with multiple organ dysfunction syndrome (MODS) is a common cause of Intensive Care Unit (ICU) mortality and morbidity. Early initiation of appropriate effective antimicrobial therapy is essential for a favorable outcome in the patient with sepsis. Cultures and serology are available only after 24 to 48 hours. In the crucial hours which determine the prognosis of the patient the physician has to depend on clinical symptoms and demographic data to aid in diagnosis and management. Using scores like APACHE II at the admission and SOFA on admission and also in their due course may help in predicting outcome. Though there are some studies on sepsis in India but use of APACHE II and SOFA scores in India have been rare. OBJECTIVES: To assess morbidity and mortality of patients with multi-organ dysfunction syndrome in sepsis. To prognosticate the patients by using defined scores like SOFA and APACHE II scores. MATERIALS AND METHODS: The study was carried out in the period of November 2010 to September 2012 and 50 patients were included in the study. The detailed history, clinical examination and all the relevant laboratory investigations were done including blood culture. In the present study the conditions were defined according to standard practice and based on relevant literature. All the patients of sepsis admitted to ICU/ emergency ward were prognosticated on the basis of APACHE II score and SOFA score. We have analysed various profiles between two groups; survivor group which include the patients who are successfully discharged after recovery and nonsurvivor group which include the patients who died. RESULTS: The clinical profile of 50 patients with sepsis with MODS was studied. There were 28 males and 22 females in this cohort. In this study, 18 patients died and 32 patients survived with mortality rate of $36 \%$. In this study, though APACHE II score was high among non survivors than survivors (23.28 v/s 18.75), it was of just statistically significantly with $\mathrm{P}=0.068+$. SOFA score has been validated extensively for prognostication. In this study, extensive study of SOFA score was done from day 1 to the last day. The SOFA score on day 1 was high among non survivors and survivors which was statistically significant (10.17 v/s 7.94, p=0.014). However the most significant difference was observed on Day 3. The SOFA score was very high among non survivors as compared to survivors which was statistically very significant.(13.42 v/s 6.84, p<0.001). CONCLUSION: Serial measurement of SOFA score during first week is very useful tool in predicting the outcome especially on the day 3 . The trend of SOFA score was progressively declining in survivors while non-survivors had stable higher score during the first week. The APACHE II score on day of admission, though reliable, was not effective in predicting the mortality rate in our set up.

KEY WORDS: APACHE II, SOFA, MODS, SURVIVORS, NON-SURVIVORS 
INTRODUCTION: Though the term sepsis is linked closely to modern intensive care, the medical concept is rather old. The word sepsis is derived from the Greek word sipsi meaning: "make rotten". ${ }^{1}$

The cause for sepsis was found only in recent times. It was Ignaz Semmelweis (1818-1865) who first deducted that fever was caused by decomposed animal matter that entered the blood system. $^{2}$ Louis Pasteur (1822-1895) soon identified microbes as single celled organisms that cause putrefaction.

One of the first attempts to establish a set of clinical parameters to define patients who have severe sepsis came in 1989 when Roger Bone and colleagues proposed the term "sepsis syndrome." 3 Following on from the sepsis syndrome; in 1991, the American College of Chest Physicians/Society of Critical Care Medicine Consensus Panel developed definitions of the various stages of sepsis. ${ }^{4}$

\begin{tabular}{|c|c|}
\hline Bacteremia & $\begin{array}{c}\text { Presence of bacteria in blood, as evidenced by } \\
\text { positive blood Cultures }\end{array}$ \\
\hline Septicemia Presence & Presence of microbes or toxins in blood \\
\hline $\begin{array}{l}\text { Systemic Inflammatory Response } \\
\text { syndrome (SIRS) }\end{array}$ & $\begin{array}{l}\text { Two or more of the following conditions: } \\
\left.\text { 1. fever (oral temperature }>38^{\circ} \mathrm{C}\right) \text { or } \\
\text { hypothermia }\left(<36^{\circ} \mathrm{C}\right) \\
\text { 2. tachypnea }(>24 \text { breaths } / \mathrm{min}) \text {; } \\
\text { 3. tachycardia (heart rate }>90 \text { beats } / \text { min); } \\
\text { 4. leukocytosis }(>12,000 / \mu \mathrm{L}) \text {, leukopenia }(<4 \text {, } \\
000 / \mu \mathrm{L}) \text {, or }>10 \% \text { bands; may have a } \\
\text { noninfectious etiology }\end{array}$ \\
\hline Sepsis & $\begin{array}{l}\text { SIRS that has a proven or suspected microbial } \\
\text { etiology }\end{array}$ \\
\hline $\begin{array}{l}\text { Severe sepsis } \\
\text { (similar to "sepsis syndrome") }\end{array}$ & $\begin{array}{l}\text { Sepsis with one or more signs of organ } \\
\text { dysfunction-for example: } \\
\text { 1. Cardiovascular: Arterial systolic blood } \\
\text { pressure } \leq 90 \mathrm{~mm} \mathrm{Hg} \text { or mean arterial } \\
\text { pressure } \leq 70 \mathrm{~mm} \mathrm{Hg} \text { that responds to } \\
\text { administration of intravenous fluid } \\
\text { 2. Renal: Urine output<0.5 } \mathrm{mL} / \mathrm{kg} \text { per hour for } \\
1 \text { hour despite adequate fluid resuscitation } \\
\text { 3. Respiratory: PaO2/FIO2 } 250 \text { or, if the lung is } \\
\text { the only dysfunctional organ, } \leq 200 \\
\text { 4. Hematologic: Platelet count }<80,000 / \mu \mathrm{L} \text { or } \\
\text { 50\% decrease in platelet count from highest } \\
\text { value recorded over previous } 3 \text { days } \\
\text { 5. Unexplained metabolic acidosis: A pH } 7.30 \\
\text { or a base deficit } 5.0 \mathrm{mEq} / \mathrm{L} \text { and a plasma } \\
\text { lactate level }>1.5 \text { times upper limit of normal } \\
\text { for reporting lab. } \\
\text { 6. Adequate fluid resuscitation: Pulmonary } \\
\text { artery wedge pressure } 12 \mathrm{~mm} \text { Hg or central } \\
\text { venous pressure } 8 \mathrm{~mm} \mathrm{Hg}\end{array}$ \\
\hline Septic shock & Sepsis with hypotension (arterial blood pressure \\
\hline
\end{tabular}




\begin{tabular}{|c|c|}
\hline & $\begin{array}{l}<90 \mathrm{~mm} \text { Hg systolic, or } 40 \mathrm{mmHg} \text { less than } \\
\text { patient's normal blood pressure) for at least } 1 \\
\text { hour despite adequate fluid resuscitation; or } \\
\text { need for vasopressors to maintain systolic blood } \\
\text { pressure } \geq 90 \mathrm{~mm} \text { Hg or mean arterial pressure } \\
\geq 70 \mathrm{~mm} \mathrm{Hg}\end{array}$ \\
\hline Refractory septic Shock & $\begin{array}{l}\text { Septic shock that lasts for }>1 \text { hour and does not } \\
\text { respond to fluid or pressor administration }\end{array}$ \\
\hline
\end{tabular}

In India tropical infections causing multiple organ dysfunction add to the burden of sepsis in ICU. Most patients present with acute undifferentiated fever with clinical syndromes like such as fever-myalgia, fever-arthralgia, fever-icterus, fever-rash, or acute encephalitic syndrome. ${ }^{5}$ Due to their varied presentation, multi system involvement and lack of clinical diagnostic criteria these tropical infections are often undiagnosed. The lack of sensitive tests to identify these infections, high cost and non availability of isolation techniques, add to the diagnostic dilemma. ${ }^{6}$

There is a need to identify the common tropical infections contributing to mortality in ICU. Studies in India have focused on patients with sepsis due to established causes like malaria, leptospirosis or rickettsial infections. There are very few studies done to study the clinical course in patients presenting with acute undifferentiated fever. ${ }^{7}$ When a patient is admitted in ICU the aetiology is usually not established. The intensivists have very little data to treat such patients in the first 24 to 48 hours which are crucial in reversing the process of sepsis and multi organ dysfunction. There are many scoring systems which are helpful in prognosticating the severity and outcome. But our study focuses on mainly Acute Physiology and Chronic Health Evaluation II (APACHE II) and Sequential organ failure assessment (SOFA) scores.

\section{OBJECTIVES:}

- To assess morbidity and mortality of patients with multi-organ dysfunction syndrome in sepsis.

- To prognosticate the patients by using defined scores like SOFA and APACHE II scores.

\section{MATERIALS AND METHODOLOGY: INCLUSION CRITERIA}

- Patients above 18 years of age

- Patients with evidence of sepsis and MODS on admission

\section{EXCLUSION CRITERIA}

- Patients who is on treatment with immunosuppressive agents

- Patients with retroviral infection

- Pregnant patients

- A prospective study entitled "USEFULNESS OF SOFA AND APACHE II SCORE IN ANALYSING PATIENTS WITH MULTIPLE ORGAN DYSFUNCTION SYNDROME IN SEPSIS” as undertaken at KIMS Hospital, Bangalore 
- The study was carried out in the period of November 2010 to September 2012 and 50 patients were included in the study.

- The patients with sepsis as defined by the American College of Chest Physicians/Society of Critical Care Medicine (ACCP/SCCM) Consensus Committee in 1992 were included in the study.

- The detailed history, clinical examination and all the relevant laboratory investigations were done including blood culture. In the present study the conditions were defined according to standard practice and based on relevant literature.

- All the patients of sepsis admitted to ICU/ emergency ward are being prognosticated on the basis of APACHE II score and SOFA score.

- APACHE II is calculated on day of admission. The predicted mortality rate was calculated on the basis of this score.

- To assess sequential involvement of organ we calculated SOFA score on every day. This gave us idea whether involvement of number of organ was increasing or decreasing and if the severity of particular organ was increasing.

- The minimum SOFA score was 0 and maximum of 24 .

- We are analyzing various profiles between two groups; survivor group which include the patients who are successfully discharged after recovery and non-survivor group which include the patients who died.

- SOFA and APACHE II charts are as follows

\begin{tabular}{|c|c|c|c|c|c|c|c|c|c|c|}
\hline \multirow[t]{2}{*}{ Physiologic Variable } & \multicolumn{5}{|c|}{ High Abnormal Range } & \multicolumn{5}{|c|}{ Low Abnormal Range } \\
\hline & +4 & +3 & +2 & +1 & 0 & +1 & +2 & +3 & +4 & Points \\
\hline $\begin{array}{l}\text { Temperature - rectal } \\
\left({ }^{\circ} \mathrm{C}\right)\end{array}$ & $\geq 41^{\circ}$ & $\begin{array}{l}39 \text { to } \\
40.9^{\circ} \\
\end{array}$ & & $\begin{array}{l}38.5 \text { to } \\
38.9^{\circ} \\
\end{array}$ & $\begin{array}{l}36 \text { to } \\
38.4^{\circ} \\
\end{array}$ & $\begin{array}{l}34 \text { to } \\
35,9^{\circ}\end{array}$ & $\begin{array}{l}32 \text { to } \\
33.9^{\circ}\end{array}$ & $\begin{array}{l}30 \text { to } \\
31.9^{\circ}\end{array}$ & $\leq 29.9^{\circ}$ & \\
\hline $\begin{array}{l}\text { Mean Arterial Pressure } \\
-\mathrm{mm} \mathrm{Hg}\end{array}$ & $\geq 160$ & \begin{tabular}{|l|}
130 to \\
159
\end{tabular} & $\begin{array}{l}110 \text { to } \\
129\end{array}$ & & $\begin{array}{l}70 \text { to } \\
109\end{array}$ & & $\begin{array}{l}50 \text { to } \\
69\end{array}$ & & $\leq 49$ & \\
\hline $\begin{array}{l}\text { Heart Rate (ventricular } \\
\text { response) }\end{array}$ & $\geq 180$ & \begin{tabular}{|l}
140 to \\
179
\end{tabular} & $\begin{array}{l}110 \text { to } \\
139\end{array}$ & & $\begin{array}{l}70 \text { to } \\
109\end{array}$ & & $\begin{array}{l}55 \text { to } \\
69\end{array}$ & $\begin{array}{l}40 \text { to } \\
54\end{array}$ & $\leq 39$ & \\
\hline $\begin{array}{l}\text { Respiratory Rate } \\
\text { (non-ventilated or } \\
\text { ventilated) }\end{array}$ & $\geq 50$ & $\begin{array}{l}35 \text { to } \\
49\end{array}$ & & $\begin{array}{l}25 \text { to } \\
34\end{array}$ & $\begin{array}{l}12 \text { to } \\
24\end{array}$ & $\begin{array}{l}10 \text { to } \\
11\end{array}$ & 6 to 9 & & $\leq 5$ & \\
\hline $\begin{array}{l}\text { Oxygenation: } \mathrm{A}-\mathrm{aDO} 2 \\
\text { or } \mathrm{PaO} 2(\mathrm{~mm} \mathrm{Hg}) \\
\text { a. } \mathrm{FlO} 2 \geq 0.5 \text { record } \\
\mathrm{A}-\mathrm{aDO} 2 \\
\text { b. } \mathrm{FlO}_{2}<0.5 \text { record } \\
\mathrm{PaO2}\end{array}$ & $\geq 500$ & \begin{tabular}{|l}
350 to \\
499
\end{tabular} & $\begin{array}{l}200 \text { to } \\
349\end{array}$ & & P02>70 & $\begin{array}{l}P O 2 \\
61 \text { to } \\
70\end{array}$ & & $\begin{array}{l}\mathrm{PO} 2 \\
55 \text { to } \\
60\end{array}$ & $P O 2<55$ & \\
\hline $\begin{array}{l}\text { Arterial pH (preferred) } \\
\text { Serum } \mathrm{HCO} 3 \text { (venous } \\
\text { mEq/) } \\
\text { (not preferred, but } \\
\text { may use if no } A B G s \text { ) }\end{array}$ & $\geq 7.7$ & $\begin{array}{l}7.6 \text { to } \\
7.69 \\
41 \text { to } \\
51.9\end{array}$ & & $\begin{array}{l}7.5 \text { to } \\
7.59 \\
32 \text { to } \\
40.9\end{array}$ & $\begin{array}{l}7.33 \text { to } \\
7.49 \\
22 \text { to } \\
31.9\end{array}$ & & $\begin{array}{l}7.25 \\
\text { to } \\
7.32 \\
18 \text { to } \\
21.9\end{array}$ & \begin{tabular}{l|}
7.15 \\
to \\
7.24 \\
\\
15 to \\
17.9 \\
\end{tabular} & $\begin{array}{l}<7,15 \\
<15\end{array}$ & \\
\hline Serum Sodium (mEg/l) & $\geq 180$ & $\begin{array}{l}160 \text { to } \\
179\end{array}$ & \begin{tabular}{|l|}
155 to \\
159
\end{tabular} & $\begin{array}{l}150 \text { to } \\
154\end{array}$ & $\begin{array}{l}130 \text { to } \\
149\end{array}$ & & $\begin{array}{l}120 \text { to } \\
129\end{array}$ & $\begin{array}{l}111 \text { to } \\
119\end{array}$ & $\leq 110$ & \\
\hline $\begin{array}{l}\text { Serum Potassium } \\
(\mathrm{mEg} / \mathrm{l})\end{array}$ & $\geq 7$ & $\begin{array}{l}6 \text { to } \\
6.9\end{array}$ & & $\begin{array}{l}5.5 \text { to } \\
5.9\end{array}$ & $\begin{array}{l}3.5 \text { to } \\
5.4\end{array}$ & $\begin{array}{l}3 \text { to } \\
3.4\end{array}$ & $\begin{array}{l}2.5 \text { to } \\
2.9\end{array}$ & & $<2,5$ & \\
\hline $\begin{array}{l}\text { Serum Creatinine } \\
(\mathrm{mg} / \mathrm{d}) \\
\text { Double point score for } \\
\text { acute renal failure }\end{array}$ & $\geq 3.5$ & $\begin{array}{l}2 \text { to } \\
3.4\end{array}$ & $\begin{array}{l}1.5 \text { to } \\
1.9\end{array}$ & & $\begin{array}{l}0.6 \text { to } \\
1.4\end{array}$ & & $<0.6$ & & & \\
\hline Hematocit (\%) & $\geq 60$ & & $\begin{array}{l}50 \text { to } \\
59.9\end{array}$ & \begin{tabular}{|l|}
46 to \\
49.9 \\
\end{tabular} & $\begin{array}{l}30 \text { to } \\
45.9\end{array}$ & & $\begin{array}{l}20 \text { to } \\
29.9\end{array}$ & & $<20$ & \\
\hline $\begin{array}{l}\text { White Blood Count } \\
\text { (total/mm3) } \\
\text { (in 1000s) }\end{array}$ & $\geq 40$ & & $\begin{array}{l}20 \text { to } \\
39.9\end{array}$ & $\begin{array}{l}15 \text { to } \\
19.9\end{array}$ & $\begin{array}{l}3 \text { to } \\
14.9\end{array}$ & & $\begin{array}{l}1 \text { to } \\
2,9\end{array}$ & & $<1$ & \\
\hline $\begin{array}{l}\text { Glasgow Coma Score } \\
\text { (GCS) } \\
\text { Score }=15 \text { minus } \\
\text { actual GCS }\end{array}$ & & & & & & & & & & \\
\hline \multicolumn{10}{|c|}{ A. Total Acute Physiology Score (sum of 12 above points) } & \\
\hline \multirow{2}{*}{\multicolumn{10}{|c|}{$\begin{array}{l}\text { B. Age points (years) } \leq 44=0 ; 45 \text { to } 54=2 ; 55 \text { to } 64=3 ; 65 \text { to } 74=5 ; \geq 75=6 \\
\text { C. Chronic Health Points (see below) }\end{array}$}} & \\
\hline & & & & & & & & & & \\
\hline \multicolumn{10}{|c|}{$\begin{array}{l}\text { C. Chronic Health Points (see below) } \\
\text { Total APACHE II Score (add together th }\end{array}$} & \\
\hline
\end{tabular}

APACHE II 


\section{ORIGINAL ARTICLE}

\section{C chronic health points:}

If the patient has a history of severe organ system insufficiency or is immunocompromised assign points as follows:

\footnotetext{
a. For nonoperative or emergency postoperative patients - 5 points

b. For elective postoperative patients -2 points
}

Chronic Diagnosis/ organ system insufficiency includes biopsy proven cirrhosis and documented portal hypertension; past upper GI bleeding attributed to portal hypertension; prior hepatic failure; prior hepatic encephalopathy; NYHA class IV; chronic restrictive, obstructive, or vascular lung disease resulting in severe exercise restriction; documented hypoxemia or hypercapnia; secondary polycythemia; severe pulmonary hypertension ( $>40 \mathrm{~mm} \mathrm{Hg}$ ); ventilator dependence; chronic hemodialysis.

Chronic Diagnosis also includes immunosuppression from chemotherapy, radiation therapy, longterm or recent high-dose steroids, immunodeficiency (eg, leukemia, lymphoma, AIDS).

\begin{tabular}{|c|c|c|c|c|c|}
\hline \multirow[b]{2}{*}{ Variables } & \multicolumn{5}{|c|}{ SOFA Score } \\
\hline & 0 & 1 & 2 & 3 & 4 \\
\hline $\begin{array}{l}\text { Respiratory } \\
\mathrm{PaO}_{2} / \mathrm{FiO}_{2}, \mathrm{~mm} \mathrm{Hg}\end{array}$ & $>400$ & $\leq 400$ & $\leq 300$ & $\leq 200 \dagger$ & $\leq 100 \dagger$ \\
\hline $\begin{array}{l}\text { Coagulation } \\
\text { Platelets } \times 10 \% / 4 L \neq\end{array}$ & $>150$ & $\leq 150$ & $\leq 100$ & $\leq 50$ & $\leq 20$ \\
\hline $\begin{array}{l}\text { Liver } \\
\text { Birubin, mg/dLf }\end{array}$ & $<1.2$ & $1.2-1.9$ & $2.0-5.9$ & $6.0-11.9$ & $>12.0$ \\
\hline $\begin{array}{l}\text { Cardiovascular } \\
\text { Hypotension }\end{array}$ & No hypotension & $\begin{array}{l}\text { Mean arterial } \\
\text { pressure } \\
<70 \mathrm{~mm} \mathrm{Hg}\end{array}$ & $\begin{array}{l}\text { Dop } \leq 5 \text { or dob } \\
\quad \text { (any dose) }\}\end{array}$ & $\begin{array}{l}\text { Dop }>5, \text { epi } \leq 0.1 \\
\quad \text { or norepi } \leq 0.1 \S\end{array}$ & $\begin{array}{r}\text { Dop }>15, \text { epi }>0.1, \\
\quad \text { or norepi }>0.1 \S\end{array}$ \\
\hline $\begin{array}{l}\text { Central nervous system } \\
\text { Glasgow Coma Score Scale }\end{array}$ & 15 & $13-14$ & $10-12$ & 6.9 & $<6$ \\
\hline $\begin{array}{l}\text { Renal } \\
\text { Creatinine, } \mathrm{mg} / \mathrm{dL} \text {. } \\
\text { or urine output, } \mathrm{mL} / \mathrm{d} \text { | }\end{array}$ & $<1.2$ & $1.2-1.9$ & $2.0-3.4$ & $3.5-4.9 \alpha<<500$ & $>5.00 r<200$ \\
\hline 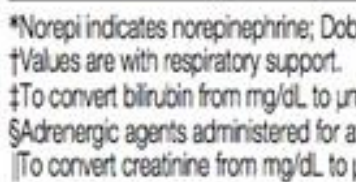 & $\begin{array}{l}\text { utarine; Dop, docam } \\
\text { multipy by } 17.1 \text {. } \\
1 \text { hour (doses given } \\
\text { Lititcy by 88.4. }\end{array}$ & $\begin{array}{l}\text { Epi, epinephrine, and } \\
\text { in vg/kg per minute). }\end{array}$ & fraction of inspiredo & & \\
\hline
\end{tabular}

Statistical Methods: Descriptive and inferential statistical analysis has been carried out in the present study. Results on continuous measurements are presented on Mean \pm SD (Min-Max) and results on categorical measurements are presented in Number (\%). Significance is assessed at $5 \%$ level of significance. The following assumptions on data is made. 
Assumptions: 1. Dependent variables should be normally distributed, 2. Samples drawn from the population should be random, Cases of the samples should be independent.

Student $t$ test (two tailed, independent) has been used to find the significance of study parameters on continuous scale between two groups Inter group analysis) on metric parameters. Leven1s test for homogeneity of variance has been performed to assess the homogeneity of variance. Chi-square/ Fisher Exact test has been used to find the significance of study parameters on categorical scale between two or more groups.

\section{RESULTS}

- The study was carried out in the period of November 2010 to September 2012 and 50 patients were included in the study.

- In the present study subjects were in the age group of 18 to 90 years.

- In the present study out of 50 cases of sepsis with MODS, 28 were male and 22 were females.

- Out of 50 patients, all 50 had fever, with breathlessness present in 16 patients.

- Co-Morbidities observed were diabetes, hypertension and COPD.

Table 1: Distribution of symptoms of patient studied

\begin{tabular}{|l|c|c|}
\hline \multicolumn{1}{|c|}{ Symptoms \& signs } & $\begin{array}{c}\text { Number of } \\
\text { patients n=50) }\end{array}$ & \% \\
\hline 1.Fever & 50 & 100.0 \\
\hline 2.Headache & 4 & 8.0 \\
\hline 3.Cough & 13 & 26.0 \\
\hline 4.Breathlessness & 16 & 32.0 \\
\hline 5.Altered Sensorium & 2 & 4.0 \\
\hline 6.Vomiting & 12 & 24.0 \\
\hline 7.Jaundice & 4 & 8.0 \\
\hline 8.Decreased Urine output & 16 & 32.0 \\
\hline 9.Abdominal Pain & 16 & 32.0 \\
\hline 10.Chest pain & 3 & 6.0 \\
\hline 11.Loose stools & 5 & 10.0 \\
\hline
\end{tabular}




\section{ORIGINAL ARTICLE}

Table 2: Comparison of comorbidities with survivors and non survivors of patients studied

\begin{tabular}{|c|c|c|c|c|}
\hline \multirow{2}{*}{ Comorbidities } & \multicolumn{4}{|c|}{ Survived } \\
\cline { 2 - 5 } & \multicolumn{2}{|c|}{ Non survived (n=18) } & \multicolumn{2}{c|}{ Survived (n=32) } \\
\cline { 2 - 5 } & No. & $\%$ & No. & $\%$ \\
\hline Nil & 8 & 44.4 & 18 & 56.2 \\
\hline Present & 10 & 55.5 & 14 & 43.7 \\
\hline$\bullet \quad$ Diabetes & 6 & 33.3 & 8 & 25.0 \\
\hline$\bullet \quad$ Hypertension & 5 & 27.8 & 3 & 9.3 \\
\hline - Paraplegic & 1 & 5.6 & 0 & 0.0 \\
\hline COPD/IHD & 2 & 11.1 & 1 & 3.1 \\
\hline
\end{tabular}

Comorbidities are statistically similar in two groups with $\mathrm{p}=0.423$

Table 3: Comparison of systemic disease with survivors and non survivors of patients studied

\begin{tabular}{|l|c|c|c|c|c|}
\hline \multirow{2}{*}{\begin{tabular}{c}
\multirow{2}{*}{$\begin{array}{c}\text { Systemic } \\
\text { disease }\end{array}$} \\
\cline { 2 - 5 }
\end{tabular}} & \multicolumn{4}{|c|}{$\begin{array}{c}\text { Non survived } \\
(\mathbf{n = 1 8})\end{array}$} & \multicolumn{2}{|c|}{$\begin{array}{c}\text { Survived } \\
(\mathbf{n}=\mathbf{3 2})\end{array}$} & \multirow{2}{*}{ p value } \\
\cline { 2 - 5 } & No. & $\%$ & No. & $\%$ & \\
\hline RS & 9 & 50.0 & 21 & 65.6 & 0.279 \\
\hline CVS & 1 & 5.6 & 2 & 6.2 & 0.921 \\
\hline CNS & 10 & 55.6 & 7 & 21.8 & $0.0169^{*}$ \\
\hline
\end{tabular}

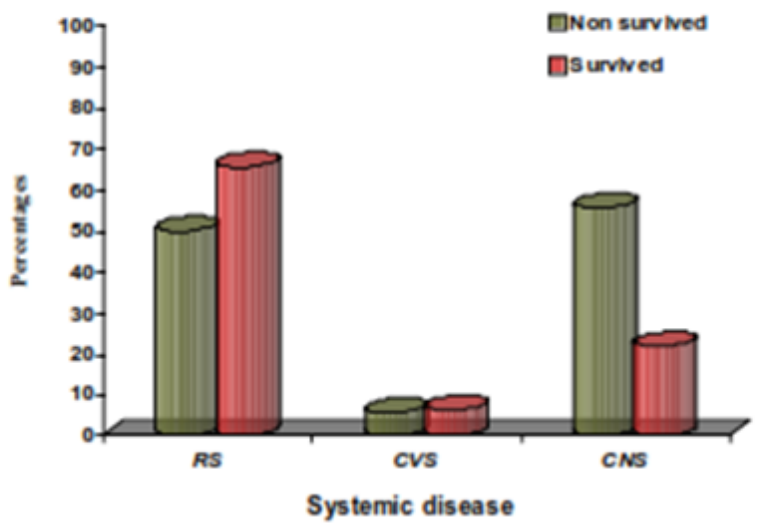




\section{ORIGINAL ARTICLE}

Table 4: Evaluation of study variables with survivors and non survivors of patients studied

\begin{tabular}{|l|c|c|c|}
\hline \multicolumn{1}{|c|}{ Variables } & Non-survived & Survived & p value \\
\hline Age in years & $51.06 \pm 19.59$ & $46.84 \pm 15.77$ & 0.411 \\
\hline Temperature & $102.48 \pm 1.06$ & $102.61 \pm 1.01$ & 0.658 \\
\hline Pulse rate & $123.44 \pm 13.51$ & $117.63 \pm 5.04$ & $0.033^{*}$ \\
\hline Respiratory rate & $27.22 \pm 5.54$ & $26.5 \pm 5.47$ & 0.658 \\
\hline Hemoglobin & $11.14 \pm 4.02$ & $10.89 \pm 2.04$ & 0.770 \\
\hline Total count & $15827.78 \pm 8423.69$ & $22893.75 \pm 24048.53$ & 0.236 \\
\hline Serum sodium & $132.83 \pm 4.79$ & $130.53 \pm 3.92$ & $0.072+$ \\
\hline Serum potassium & $4.24 \pm 0.67$ & $3.96 \pm 0.85$ & 0.236 \\
\hline PH & $7.24 \pm 0.13$ & $7.24 \pm 0.1$ & 0.989 \\
\hline Serum amylase & $20.56 \pm 11.55$ & $17.69 \pm 5.97$ & 0.251 \\
\hline Haematocrit & $37.54 \pm 9.67$ & $38.58 \pm 4.39$ & 0.605 \\
\hline
\end{tabular}

Table 5: Evaluation of GCS with survivors and non survivors of patients studied

\begin{tabular}{|l|c|c|}
\hline \multicolumn{1}{|c|}{ GCS } & Non survived & Survived \\
\hline Day 1 & $10.06 \pm 5.57$ & $14.19 \pm 1.99$ \\
\hline Day 2 & $10.5 \pm 5.22$ & $13.84 \pm 2.26$ \\
\hline Day 3 & $9.23 \pm 5.18$ & $14.03 \pm 2.09$ \\
\hline Day 4 & $9.33 \pm 5.02$ & $14.41 \pm 1.6$ \\
\hline Day 5 & $8 \pm 5.63$ & $14.5 \pm 1.55$ \\
\hline Day 6 & $7.38 \pm 5.63$ & $14.63 \pm 1.35$ \\
\hline Day 7 & $8.2 \pm 4.66$ & $14.92 \pm 0.41$ \\
\hline Day 8 & $8.2 \pm 4.66$ & $15 \pm 0$ \\
\hline Day 9 & $8.8 \pm 4.82$ & $15 \pm 0$ \\
\hline Day 10/last day & $8.67 \pm 5.13$ & $13.75 \pm 4.33$ \\
\hline
\end{tabular}


GCS among survivors and non survivors were as follows.GCS among survivors was high and statistically significant. It independently predicted the outcome and also added its value to SOFA score for prediction.

Table 6: Evaluation of serum creatinine with survivors and non survivors of patients studied

\begin{tabular}{|l|c|c|}
\hline Serum Creatinine(mg/dl) & Non survived & Survived \\
\hline Day 1 & $1.76 \pm 1.06$ & $2.77 \pm 2.43$ \\
\hline Day 2 & $2.15 \pm 1.14$ & $2.75 \pm 2.1$ \\
\hline Day 3 & $2.28 \pm 0.89$ & $2.23 \pm 1.48$ \\
\hline Day 4 & $2.36 \pm 1.22$ & $2.13 \pm 1.41$ \\
\hline Day 5 & $2.54 \pm 1.8$ & $2.17 \pm 1.46$ \\
\hline Day 6 & $2.49 \pm 1.97$ & $1.94 \pm 1.42$ \\
\hline Day 7 & $1.88 \pm 1.02$ & $1.77 \pm 1.12$ \\
\hline Day 8 & $2.4 \pm 1.12$ & $1.58 \pm 0.86$ \\
\hline Day 9 & $2.48 \pm 1.19$ & $1.46 \pm 0.73$ \\
\hline Day 10/last day & $2.58 \pm 1.56$ & $1.26 \pm 0.5$ \\
\hline
\end{tabular}

Serum creatinine among survivors and non survivors were as follows. Though, serum creatinine wasn't statistically significant between the groups, it added its value to SOFA score for prediction

Table 7: Comparison of ventilator support, dialysis, inotropic support and duration of ICU stay with survivors and non survivors of patients studied

\begin{tabular}{|l|c|c|c|}
\hline & $\begin{array}{c}\text { Non survived } \\
(\mathbf{n}=\mathbf{1 8})\end{array}$ & $\begin{array}{c}\text { Survived } \\
(\mathbf{n = 3 2})\end{array}$ & P value \\
\hline Ventilator support & $16(88.9 \%)$ & $14(43.8 \%)$ & $0.002^{* *}$ \\
\hline Dialysis & $2(11.1 \%)$ & $8(25.0 \%)$ & 0.295 \\
\hline Inotropic support & $13(72.2 \%)$ & $15(46.9 \%)$ & $0.083+$ \\
\hline $\begin{array}{l}\text { Duration of ICU } \\
\text { stay }\end{array}$ & $3.72 \pm 3.08$ & $3.75 \pm 2.02$ & 0.969 \\
\hline
\end{tabular}




\section{ORIGINAL ARTICLE}

16 out of $18(88.9 \%)$ among non survivors required ventilator support whereas 14 out of $32(43.8 \%)$ among survivors required ventilator support suggesting significant respiratory system involvement among non survivors $(\mathrm{p}=0.002)$. The mean duration of ICU stay did not vary between non-survivors and survivors (3.72 v/s 3.75$)$.

13 out of $18(72.2 \%)$ among non-survivors required inotropic support whereas 15 out of $32(46.9 \%)$ among survivors required inotropic support suggesting statistically significant hypotension among non-survivors $(\mathrm{p}=0.083)$. However, dialysis was required more among survivors than non-survivors $(25 \% \mathrm{v} / \mathrm{s} 11.1 \%, \mathrm{p}=0.295)$ but was not statistically very significant.

Serum creatinine among survivors who underwent dialysis varied between $6 \mathrm{mg} / \mathrm{dl}$ to $10 \mathrm{mg} / \mathrm{dl}$

Table 8: Comparison of SOFA score with survivors and non survivors of patients studied

\begin{tabular}{|l|c|c|c|}
\hline \multicolumn{1}{|c|}{ SOFA score } & Non survived & Survived & p value \\
\hline Day 1 & $10.17 \pm 3.45$ & $7.94 \pm 2.64$ & $0.014^{*}$ \\
\hline Day 2 & $11.63 \pm 4.33$ & $8.28 \pm 2.62$ & $0.002^{* *}$ \\
\hline Day 3 & $13.42 \pm 4.06$ & $6.84 \pm 2.96$ & $<0.001^{* *}$ \\
\hline Day 4 & $10.78 \pm 3.77$ & $5.94 \pm 3.41$ & $0.001^{* *}$ \\
\hline Day 5 & $12.25 \pm 4.8$ & $4.55 \pm 3.27$ & $<0.001^{* *}$ \\
\hline Day 6 & $12.29 \pm 6.1$ & $3.39 \pm 2.77$ & $<0.001^{* *}$ \\
\hline Day 7 & $14.2 \pm 3.9$ & $2.82 \pm 2.61$ & $<0.001^{* *}$ \\
\hline Day 8 & $13 \pm 3.39$ & $2.45 \pm 2.5$ & $<0.001^{* *}$ \\
\hline Day 9 & $13.8 \pm 4.09$ & $1.81 \pm 1.72$ & $<0.001^{* *}$ \\
\hline Day 10/last day & $13.5 \pm 5.69$ & $1.33 \pm 1.23$ & $<0.001^{* *}$ \\
\hline
\end{tabular}




\section{ORIGINAL ARTICLE}

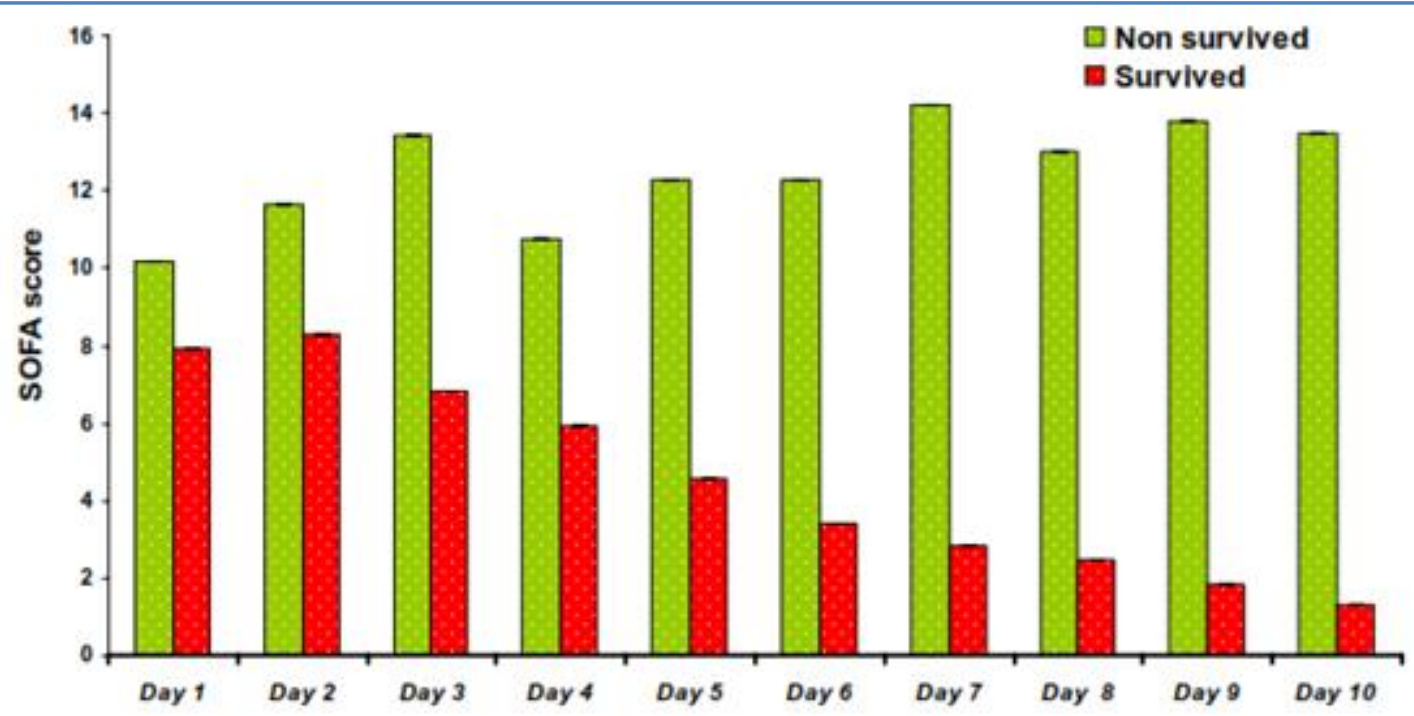

Out of 50 patients studied, SOFA score was significantly low especially on day 3

$(6.84 \pm 2.96)$ in survivor group as compared to non survivor group whose mean day 3 value being ( $13.42 \pm 4.060)$.

Table 9: Comparison of APACHE II score with survivors and non survivors of patients studied

\begin{tabular}{|l|c|c|}
\hline \multicolumn{1}{|c|}{ APACHE II } & Non survived & Survived \\
\hline$<10$ & $2(11.1 \%)$ & $4(12.5 \%)$ \\
\hline $10-20$ & $5(27.8 \%)$ & $16(50.0 \%)$ \\
\hline $20-30$ & $8(44.4 \%)$ & $10(31.3 \%)$ \\
\hline$>30$ & $3(16.7 \%)$ & $2(6.3 \%)$ \\
\hline Total & $18(100.0 \%)$ & $32(100.0 \%)$ \\
\hline Mean \pm SD & $23.28 \pm 9.65$ & $18.75 \pm 7.34$ \\
\hline
\end{tabular}




\section{ORIGINAL ARTICLE}

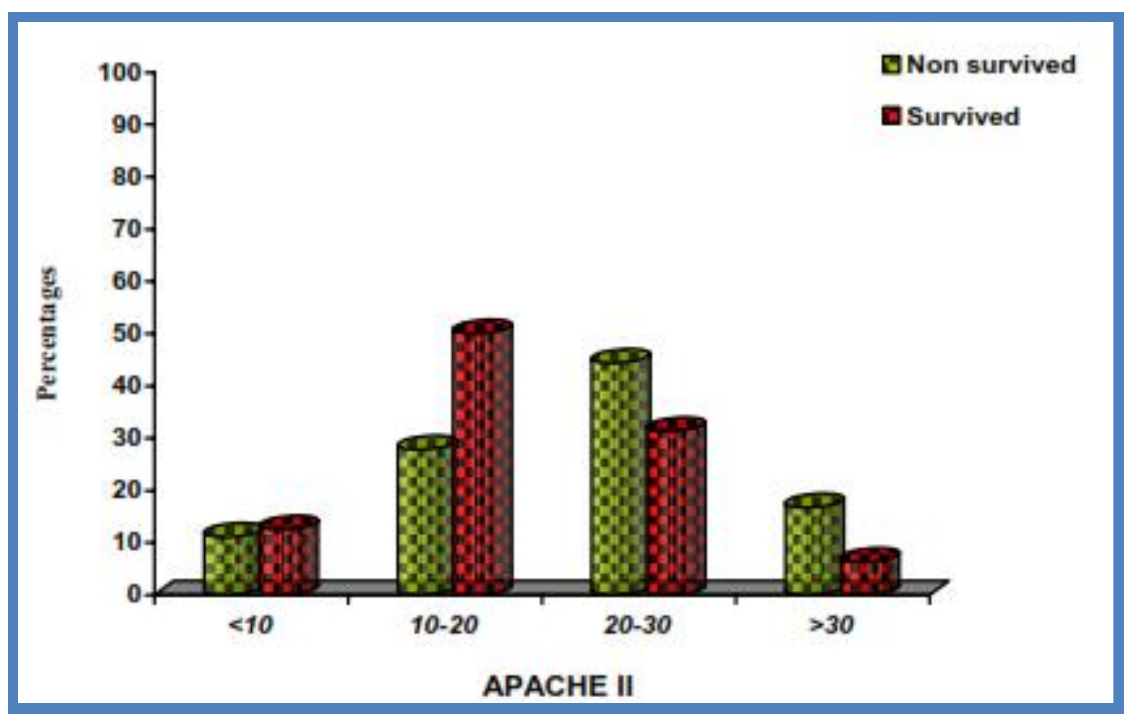

APACHE II score is significantly more in non survived patients with $\mathrm{p}=0.068+$.

DISCUSSION: The clinical profile of 50 patients with sepsis with MODS was studied. There were 28 males and 22 females in this cohort. The age of patients varied from 18 years to 90 years. The mean age was 48.36 years. Similar studies in India have shown male preponderance with most patients in the fourth to fifth decade. ${ }^{8}$ Even in this study, most patients were in fourth to fifth decade. Comorbidities were present in 24 patients with diabetes mellitus being present in 14 patients.

All patients had fever with breathlessness being the next predominant symptom observed in 16 patients. Even decreased urine output was observed in 16 patients accounting for acute kidney injury. Among the several disorders encountered in sepsis, acute kidney injury (AKI) is one of the most important because it is a life-threatening condition, increases the complexity and cost of care, and is an independent risk factor for mortality. ${ }^{9}$

The mean SOFA score on the day of admission was 8.74 and the mean APACHE II score on the day of admission was 20.14 suggesting there was significant organ dysfunction in all patients. In this study, 30 patients required ventilator support, 28 patients required ionotropes, 10 patients required dialysis. This again suggests significant organ dysfunction. The mortality recorded in this study is $36 \%$. In large clinical trials, the mortality associated with severe sepsis and septic shock ranges between $13 \%$ and $50 \% .^{10}$

Finding the cause was not the main objective of the study. However, 9 cases of dengue were identified. 2 cases of leptospirosis was observed. There was not a single case of malaria in this study. In 4 cases of UTI, organisms were isolated: 3 were caused by Eschieria coli, 1 being klebsiella species. 1 case of H1N1 was identified. 1 special case in which anti- HAV was positive. It was not sure whether hepatitis A caused sepsis or it was an incidental finding.

Clinical predictors of mortality: In this study, 18 patients died and 32 patients survived. The mean age among non survivors was little high compared to survivors (51.7 v/s 46.84) which was not statistically significant $(\mathrm{p}=0.411) .7$ patients among non-survivors and 9 patients had breathlessness which was statistically similar (p-0.532). Even co-morbidities are statistically similar in two groups 


\section{ORIGINAL ARTICLE}

with $\mathrm{P}=0.423$. Presence of pallor, icterus are statistically similar in non-survived and survived group with $\mathrm{P}=0.830$.

The non survivors had a higher pulse rate (mean $123.44 \mathrm{v} / \mathrm{s} 117.63 \mathrm{p}=0.033$ ) and a lower blood pressure and therefore a greater requirement for ionotropes compared to survivors. Septic shock is associated with a higher mortality as shown with studies in Europe. ${ }^{11}$ Degoricija et al recorded a mortality rate of $72.1 \%$ in patients with septic shock in Croatia. ${ }^{12}$ Studies in India have recorded a mortality of $59.26 \%$ in patients with severe sepsis and septic shock. ${ }^{8}$

The respiratory rate was high in non survivors than survivors (27.22 v/s 26.5) which was not statistically significant $(\mathrm{p}=0.658)$. Leukocytosis and leukopenia is often associated with mortality and normal white blood cell counts are associated with survival. ${ }^{4}, 13$ In this study however no survivors had a mean total count of $15,827 / \mu \mathrm{L}$ and survivors had a mean total count of $22,893 / \mu \mathrm{L}$ at admission. The difference was not statistically significant.

Studies have shown that the Glasgow coma scale at admission is an independent predictor of mortality. ${ }^{14,15}$ In this study, the mean GCS among survivors was high compared to non survivors on all days (day1, $14.19 \mathrm{v} / \mathrm{s} 10.19)$ and was statistically very significant $(\mathrm{p}<0.001)$.

In this study, serum creatinine did not significantly differ among non survivors and survivors on day 1 and also on initial few days (day1, $1.76 \mathrm{v} / \mathrm{s} 2.77, \mathrm{p}=0.101$ ). Even serum bilirubin was significantly different among survivors and non survivors (day $1,2.19 \mathrm{v} / \mathrm{s} 2.78, \mathrm{p}=0.375$ ).

In this study, 16 out of $18(88.9 \%)$ among non survivors required ventilator support whereas 14 out of $32(43.8 \%)$ among survivors required ventilator support suggesting significant respiratory system involvement among non survivors $(\mathrm{p}=0.002)$. The mean duration of ICU stay did not vary between non survivors and survivors (3.72 v/s 3.75). It may be attributable to early death among non survivors and early recovery among survivors.

In this study, 13 out of $18(72.2 \%)$ among non survivors required inotropic support whereas 15 out of 32(46.9\%) among survivors required inotropic support suggesting statistically significant hypotension among non survivors $(\mathrm{p}=0.083)$. However, dialysis was required more among survivors than non survivors $(25 \% \mathrm{v} / \mathrm{s} 11.1 \%, \mathrm{p}=0.295)$ but was not statistically very significant.

Many studies have shown that high APACHE II score at the time of admission was associated with high mortality. In this study, though mean APACHE II score was high among non survivors than survivors(23.28 v/s18.75), it was of just statistical significance $(\mathrm{p}=0.068+)$

SOFA score has been validated extensively for Prognostication.In this study, extensive study of SOFA score was done from day 1 to the last day. The SOFA score on day 1 was high among non survivors and survivors which was statistically significant $(10.17 \mathrm{v} / \mathrm{s} 7.94, \mathrm{p}=0.014)$.

However the most significant difference was observed on day 3. The SOFA score was very high among non survivors as compared to survivors which was statistically very significant. (13.42 $\mathrm{v} / \mathrm{s} 6.84, \mathrm{p}<0.001$ ). This was similar to many studies that have been done. Vosylius et al in their study on 117 ICU patients with sepsis showed that the changes in the severity of organ dysfunction were closely related to the outcome of the patients admitted to ICU. The SOFA score on day 3 was better compared with SOFA score on day 1 as the tool for outcome prediction. ${ }^{14}$ Vincent et al in their study in 40 ICU's in 16 countries showed that the total SOFA score increased in $44 \%$ of the nonsurvivors but in only $20 \%$ of the survivors. ${ }^{16}$ Saulius Vosylius, Jurate Sipylaite ${ }^{17}$ in Vilnius, Lithuania observed that SOFA score on day 1 and day 3 was significantly higher in non-survivors than those in survivors. Flavi Lopez Fereria; Daliana PeresBota ${ }^{18}$ in Belgium found initial SOFA score 


\section{ORIGINAL ARTICLE}

up to 9 predicted a mortality of less than 33\% while an initial SOFA score of greater than 11 predicted a mortality rate of $95 \%$.

Studies have shown that in the SOFA scores; cardiovascular, neurological, and respiratory, renal, haematological and hepatic dysfunctions were independent risk factors for mortality. ${ }^{14,16 I n}$ our study also the same have been observed as described above for respiratory, cardiovascular and neurological variables. However renal and hepatic parameters did not vary much among non survivors and survivors

\section{CONCLUSION:}

- Serial measurement of SOFA score during first week is very useful tool in predicting the outcome. The trend of SOFA score was progressively declining in survivors while non survivors had stable higher score during the first week.

- The APACHEII score on day of admission, though reliable, was not very effective in predicting the mortality rate in our set up

\section{REFERENCES:}

1. Sullivan, R. (1996) Thales to Galen: a brief journey through rational medical philosophy in ancient Greece. Part I: pre-Hippocratic medicine. Proc. R.Coll. Physicians Edinb; 26, 135-142.

2. Best M, Neuhauser, D. "Ignaz Semmelweis and the birth of infection control." Qual Saf Health Care British Medical Journal, 2004; 13: 233-234.

3. Bone, R.C., Fisher, C.J., Clemmer, T.P., Slotman, G.J., Metz, C.A. and Balk, R.A. (1989) Sepsis syndrome: A valid clinical entity. Crit. Care Med; 17, 389-393.

4. Bone RC, Balk RA, Cerra FB, Dellinger RP, Fein AM, Knaus WA, et al. Definitions for sepsis and organ failure and guidelines for the use of innovative therapies in sepsis. The ACCP/SCCM Consensus Conference Committee. American College of Chest Physicians/Society of Critical Care Medicine.Chest 1992; 101:1644-55.

5. World Health Organization, 2002. Operations Manual for the Integrated Disease Surveillance Program: India. New Delhi: WHO.

6. Manock SR, Jacobsen KH, de Bravo NB, Russell KL, Negrete M, et al. Etiology of Acute Undifferentiated Febrile Illness in the Amazon Basin of Ecuador. Am J Trop Med Hyg. 2009 Jul; 81(1):146-151.

7. Joshi R, Colford JM Jr, Reingold AL, Kalantri S. Nonmalarial Acute Undifferentiated Fever in a Rural Hospital in Central India: Diagnostic Uncertainty and Overtreatment with Antimalarial Agents. Am J Trop Med Hyg. 2008 Mar; 78(3):393-9.

8. S Todi, S Chatterjee, S Sahu and M Bhattacharyya Epidemiology of severe sepsis in India: an update Crit Care. 2010; 14(Suppl 1): P382.

9. Potential Interventions in Sepsis-Related Acute Kidney Injury cjasn.asnjournals.org.

10. Balk RA. Severe sepsis and septic shock: definitions, epidemiology, and clinical manifestations. Crit Care Clin 2000; 16:179-92.

11. Jacobson S, Johansson G, Winsö O. Primary sepsis in a university hospital in northern Sweden: a retrospective study. Acta Anaesthesiol Scand. 2004 Sep; 48(8):960-7. 


\section{ORIGINAL ARTICLE}

12. Degoricija V, Sharma M, Legac A, Gradišer M, Šefer S and Vučičevićz. Survival Analysis of 314 Episodes of Sepsis in Medical Intensive Care Unit in University Hospital: Impact of Intensive Care Unit Performance and Antimicrobial Therapy. Croat Med J. 2006 June; 47(3): 385-397.

13. Oliveira AP, Barata $\mathrm{CH}$, Murta EF, Tavares-Murta BM.Comparative study of survivor and nonsurvivor sepsis patients in a university hospital. Rev Soc Bras Med Trop. 2008 Jan-Feb; 41(1):50-4.

14. Vosylius S, Sipylaite J, IvaskeviciusJ. Sequential Organ Failure Assessment Score as the Determinant of Outcome for Patient with Severe Sepsis. Croat Med J. 2004 Dec; 45(6):715-20.

15. Bastos PG, Sun X, Wagner DP, Wu AW, Knaus WA. Glasgow coma scale score in the evaluation of outcome in the intensive care unit: findings from the Acute Physiology and Chronic Health Evaluation III study. Crit Care Med. 1993 Oct; 21(10): 1459-65.

16. Vincent J L, de Mendonça A, Cantraine F, Moreno R, Takala J, Suter PM et al. Sprung C: Use of the SOFA score to assess the incidence of organ dysfunction/failure in intensive care units: results of a multicenter, prospective study. Working group on "sepsis related problems" of the European Society of Intensive Care Medicine.Crit Care Med 1998; Nov;26(11):1793-800.

17. SauliusVosylius et al Sequential Organ Failure Assessment Score as the Determinant of Outcome for Patients with Severe Sepsis Clinic of Anesthesiology and Intensive Care, Vilnius University, Vilnius, Lithuania.

18. Ferreira, Flavio Lopeset al How Changes in Sofa Score Can Predict Outcome Critical Care Medicine: December 1999.

\section{AUTHORS:}

1. K.S. Abhinandan

2. R. Vedavathi

\section{PARTICULARS OF CONTRIBUTORS:}

1. Senior Resident, Department of General Medicine, Kempegowda Institute of Medical Science.

2. Professor, Department of General Medicine, Kempegowda Institute of Medical Science.

\section{NAME ADRRESS EMAIL ID OF THE} CORRESPONDING AUTHOR:

Dr. K.S. Abhinandan,

Near Jayashree Nursing Home,

K.R. Puram, Hassan - 573201.

Email -abhi_310@yahoo.com

Date of Submission: 13/11/2013.

Date of Peer Review: 14/11/2013.

Date of Acceptance: 25/11/2013.

Date of Publishing: 04/12/2013 